\title{
Article
}

\section{Curcumin administration suppresses collagen synthesis in the hearts of rats with experimental diabetes}

\author{
Shuang GUO\#, Xiang-wen MENG\#, Xiao-song YANG, Xiu-fen LIU, Chang-han OU-YANG* , Chao LIU* \\ Hubei Key Laboratory of Cardiovascular, Cerebrovascular, and Metabolic Disorders, Hubei University of Science and Technology, \\ Xianning 437100, China
}

\begin{abstract}
Cardiac fibrosis is considered the initial change of diabetic cardiomyopathy (DCM). We have shown that curcumin alleviates collagen deposition in DCM, but the mechanism remains unknown. In this study we sought to investigate the effects of curcumin on cardiac fibrosis in vivo and in vitro and to elucidate the underlying mechanisms. Experimental diabetes was induced in rats by injection of lowdose streptozotocin (STZ) combined with high energy diet. The rats were orally treated with curcumin $\left(300 \mathrm{mg} \cdot \mathrm{kg}^{-1} \cdot \mathrm{d}^{-1}\right)$ for $16 \mathrm{weeks}$. Curcumin administration significantly suppressed the deposition of type I and type III collagens in the heart tissues of diabetic rats, accompanied by markedly reduced TGF- $\beta 1$ production, suppressed T $\beta R$ II levels and Smad2/3 phosphorylation, and increased Smad7 expression. Similar effects were observed in human cardiac fibroblasts exposed to high glucose (HG, 30 mmol/L) or exogenous TGF$\beta 1(5 \mathrm{ng} / \mathrm{mL})$. Furthermore, TGF- $\beta 1$ or HG treatment significantly increased the phosphorylation levels of AMPK and p38 MAPK in the fibroblasts. Application of curcumin ( $25 \mu \mathrm{mol} / \mathrm{L}$ ) inhibited TGF- 31 - or HG-induced AMPK/p38 MAPK activation and suppressed collagen synthesis in the fibroblasts. These effects were similar to those of the AMPK inhibitor compound C (10 $\mu \mathrm{mol} / \mathrm{L})$ but opposite to the effects of the AMPK activator metformin ( $2 \mathrm{mmol} / \mathrm{L}$ ) in the fibroblasts. Our results demonstrate that curcumin suppresses diabetesassociated collagen synthesis in rat myocardium not only by inhibiting TGF- $\beta 1$ production and canonical Smad signaling but also by blocking the non-canonical AMPK/p38 MAPK pathway.
\end{abstract}

Keywords: cardiac fibrosis; diabetes; curcumin; TGF- $\beta$; Smad signaling; AMPK/p38 MAPK; compound C; metformin

Acta Pharmacologica Sinica (2018) 39: 195-204; doi: 10.1038/aps.2017.92; published online 14 Sep 2017

\section{Introduction}

Diabetic cardiomyopathy (DCM) has attracted serious attention because of its increased morbidity and mortality rates within the diabetic population in recent decades. Cardiac fibrosis, a common pathological feature in the development of various cardiovascular diseases ${ }^{[1]}$, is considered the initial change of DCM. Fibrosis is generally characterized by excessive extracellular matrix (ECM) accumulation. Collagen types I and III are the main components of ECM in myocardium. They form a complex fibrillar collagen network that acts as a scaffold for cardiomyocytes. Under physiological conditions, the production and degradation of ECM is in a state of dynamic equilibrium. However, some pathological factors, such as high glucose, high fat, and oxidative stress, may stim-

\footnotetext{
"These authors contributed equally to this work.

${ }^{*}$ To whom correspondence should be addressed.

E-mail tnbsys_liuchao@126.com (Chao LIU); ouyangcch@163.com (Chang-han OU-YANG)

Received 2017-02-12 Accepted 2017-06-05
}

ulate cardiac fibroblasts to overproduce collagen and result in the excessive accumulation of ECM. Myocardial fibrosis induces interstitial remodeling and structural disorder. Ultimately, excessive ECM deposition impairs cardiac function, elicits arrhythmia, and leads to heart failure ${ }^{[2]}$.

It is known that a network of growth factors and hormones are responsible for initiating and maintaining fibrotic responses in vivo. One of the most important cytokines is transforming growth factor $\beta 1$ (TGF- $\beta 1$ ). In the canonical TGF- $\beta$ /Smad pathway, TGF- $\beta 1$ binds to and activates membrane-bound TGF- $\beta$ type II and type I receptors (T $\beta$ R II and I) that exist in heteromeric complexes. T $\beta R$ II phosphorylates T $\beta R$ I, which in turn phosphorylates Smad2 and Smad3. Phosphorylated Smad2 and 3 bind to Smad 4 and translocate into the nucleus to activate fibrogenic gene transcription. Smad7 inhibits signal transduction through this pathway by suppressing Smad2/3 phosphorylation ${ }^{[3]}$. The pro-fibrotic actions of TGF- $\beta$ also involve Smad-independent pathways. TGF- $\beta$ activates noncanonical signaling pathways that modify 
fibrogenic gene expression in a promoter-specific fashion, including pathways that involve the mitogen-activated protein kinases c-Jun N-terminal kinase, extracellular regulated protein kinases and p38.

AMP-activated protein kinase (AMPK) is a constitutively expressed serine/threonine kinase that senses cellular energy status and coordinates global metabolic responses to energy deprivation $^{[4]}$. AMPK contains a catalytic a-subunit and two regulatory subunits, $\beta$ and $\gamma$. AMPK activation requires phosphorylation of the $\alpha$-subunit on the threonine-172 residue in its activation loop ${ }^{[5]}$. In addition to regulating glucose and lipid metabolism, AMPK influences many biological processes including cytokine production, inflammation, proliferation and apoptosis. Accumulated evidence has shown that AMPK influences fibrotic progression in several systems and disease states $^{[6,7,8]}$, especially in cardiac fibrosis associated with ischemia, hypertrophy, aging and metabolic diseases, based on cross-talk with critical fibrotic signaling pathways involving TGF- $\beta^{[9]}$.

Curcumin is a naturally occurring polyphenol isolated from the plant Curcuma longa L. Experimental data in the last few decades have started to characterize this yellow bioactive agent, which possesses a wide range of physiological and pharmacological properties, such as antioxidant, anti-inflammatory, anticancer ${ }^{[10]}$, and anti-diabetic activity ${ }^{[1]]}$. Our previous studies demonstrated that curcumin alleviated myocardial collagen and improved heart function in experimental diabetic rats displaying cardiomyopathy ${ }^{[12]}$. However, the underlying molecular mechanisms remained unknown. Therefore, the present study was designed to investigate the effects of curcumin on diabetes-associated cardiac fibrosis, both in experimental diabetic rats and in human cardiac fibroblasts. As previous data from our experiments and other laboratories indicated that AMPK was involved in multiple actions of $\operatorname{curcumin}^{[13]}$, we also explored the potential roles of AMPK in mediating the effect of curcumin on collagen synthesis in cultured cardiac fibroblasts.

\section{Materials and methods Reagents}

Human cardiac fibroblasts (HCFs) and culture medium [including fibroblast medium-2 (FM-2), fetal bovine serum, FGS-2 and penicillin-streptomycin] were purchased from ScienCell (San Diego, CA, USA). Human recombinant TGF$\beta 1$ was purchased from R\&D SYSTEMS (Minneapolis, MN, USA). A human TGF- $\beta 1$ precoated ELISA kit was purchased from Dakewe (Shenzhen, China). Antibodies against collagen I, collagen III, and T $\beta R$ II were purchased from Abcam (Cambridge, MA, USA). Antibodies against phosphoSmad2 (Ser465/467)/3 (Ser423/425), Smad2/3, phosphoAMPKa (Thr172), phospho-p38 MAPK (Thr180/Tyr182), T $\beta R$ I, AMPKa and p38 MAPK were purchased from Cell Signaling Technology (Boston, MA, USA). Antibodies against TGF- $\beta 1$ and Smad7 were purchased from Santa Cruz Biotechnology (Santa Cruz, CA, USA). Total RNA extraction reagent, a Hiscript II 1st Strand cDNA Synthesis Kit, and 2× Taq Plus Master Mix were purchased from Vyzame (Nanjing, China). Diethylpyrocarbonate (DEPC) water was purchased from Beyotime Biotechnology (Shanghai, China). Biowest agarose was purchased from Gene Company Ltd (Hongkong, China). Curcumin, streptozotocin (STZ) and other reagents were purchased from Sigma (St Louis, $\mathrm{MO}, \mathrm{USA}$ ) unless noted otherwise.

\section{Animals}

Eighty male Sprague-Dawley (SD) rats were purchased from the Laboratory Animal Center of Wuhan University (SCXY (E) 2010-0009). All rats were raised according to the Guide for the Care and Use of Laboratory Animals published by the US National Institutes of Health (NIH Publication № 85-23, revised 1996). The rats were given access to water and food ad libitum and kept under a consistent daily light cycle, with $12 \mathrm{~h}$ of light and $12 \mathrm{~h}$ of darkness. All experimental operations were permitted by the Committee of Experimental Animals of Hubei University of Science and Technology. All efforts were made to minimize animal suffering.

\section{Generation of experimental diabetic rat model}

Experimental diabetic rats were given a high-glucose (10\%) and high-fat (20\%) diet for 4 weeks before receiving intraperitoneal injections of STZ ( $35 \mathrm{mg} / \mathrm{kg}$ ) for five consecutive days. STZ was dissolved in $100 \mathrm{mmol} / \mathrm{L}$ citrate buffer $(\mathrm{pH}=4.2-4.5)$ just before use. Control rats were fed a normal diet and injected intraperitoneally with an equivalent volume of citrate buffer. After 2 weeks, rats with random blood glucose levels $\leq 16.7 \mathrm{mmol} / \mathrm{L}$ were excluded from the next experiment. The remaining experimental rats were then randomly divided into 4 groups: the Con group (non-diabetic rats not treated with curcumin), the Cur group (non-diabetic rats treated with curcumin), the DM group (diabetic rats not treated with curcumin), and the DM+Cur group (diabetic rats treated with curcumin). Curcumin $\left(300 \mathrm{mg} \cdot \mathrm{kg}^{-1} \cdot \mathrm{d}^{-1}\right)$ was orally administered in the drinking water for 16 weeks. The Con and DM groups were treated with an equal volume of saline.

\section{Cell culture and treatment}

Human cardiac fibroblasts (HCFs) were cultured in FM medium-2 supplemented with 5\% FBS, penicillin and streptomycin and FGS-2. All cells were incubated at $37^{\circ} \mathrm{C}$ in a humidified atmosphere of $5 \% \mathrm{CO}_{2}$ and $95 \%$ air. Confluent cells were incubated in normal glucose (Con: $D$-glucose 5 $\mathrm{mmol} / \mathrm{L}$ ), high glucose (HG: $D$-glucose $30 \mathrm{mmol} / \mathrm{L}$ ), or human recombinant TGF- $\beta 1(5 \mathrm{ng} / \mathrm{mL})$. Curcumin $(25 \mu \mathrm{mol} / \mathrm{L})$, Compound C (CC, $10 \mu \mathrm{mol} / \mathrm{L}$ ) or metformin (Met, $2 \mathrm{mmol} / \mathrm{L}$ ) was applied to the cells for $24 \mathrm{~h}$.

\section{Masson's trichrome staining}

All rats were sacrificed after the experiment. The hearts were excised and immediately placed in $4 \%$ paraformaldehyde for paraffin embedding. The heart tissues were sectioned at a thickness of $3 \mu \mathrm{m}$ in the middle of the left ventricles along the short axis of the heart. Three to five sections per heart, separated by a distance of 50-60 $\mu \mathrm{m}$, were chosen for Mas- 
son's trichrome staining. Fibrotic tissue was stained blue, in contrast to the red-stained myocardium. The stained sections were photographed using an Olympus BX53 camera (Tokyo, Japan) by light microscopy at $\times 400$ magnification. The areas of myocardial fibrosis were quantified using Image-Pro Plus 6.0 (Media Cybernetics, Rockville, MD, USA). The sum of 5 fields per rat ( $n=3$ to 5 rats) was used for statistical analysis to test for significant differences.

\section{Immunohistochemical measurement}

Tissue sections were incubated with rabbit anti-collagen I (1:200, Abcam), rabbit anti-collagen III (1:200, Abcam) and rabbit anti-TGF- $\beta 1$ (1:50, Santa Cruz) at $4{ }^{\circ} \mathrm{C}$ overnight. After being washed 3 times with PBS, the sections were incubated with horseradish peroxidase-conjugated secondary goat antirabbit IgG (Goodbio, China) at room temperature for $60 \mathrm{~min}$. The sections were then stained with DAB under a microscope, counterstained with hematoxylin, dehydrated in a graded ethanol series, and mounted with Permount Mounting Medium. The positive areas were stained brown. Images $(\times 400$ magnification) were captured using an Olympus BX53 camera (Tokyo, Japan) and quantified using Image-Pro Plus 6.0 (Media Cybernetics, Rockville, MD, USA). The sum of 5 fields per mouse ( $n=3$ to 5 mice) was used for statistical analysis to test for significant differences.

\section{Measurement TGF- $\beta 1$ in culture medium}

TGF- $\beta 1$ secreted in the culture medium was measured using the human TGF- $\beta 1$ precoated ELISA kit (Dakewe Bio-engineering Co, Ltd, Shenzhen). The procedure was carried out according to the manufacturer's instructions.

\section{Western blotting analysis}

The total protein extracted from cells and tissues was measured using a method used previously in our laboratory ${ }^{[14]}$. Briefly, the tissues or cells were lysed in RIPA buffer supplemented with $1 \times$ protease inhibitor cocktail (Sigma-Aldrich, St Louis, MO, USA). The lysates were centrifuged at $12000 \times g$ at $4^{\circ} \mathrm{C}$ for $15 \mathrm{~min}$. The protein concentration was measured using the BCA method. The proteins were denatured in Laemmli sample buffer for $5 \mathrm{~min}$ at $95^{\circ} \mathrm{C}$, electrophoresed on $8 \%-10 \%$ SDS-polyacrylamide gel and transferred to polyvinylidene fluoride (PVDF) membranes (Millipore, USA). The membranes were blocked with 5\% skim milk prepared with $0.1 \%$ TWEEN-20 Tris-buffered saline (TBST) solution for $1 \mathrm{~h}$ at room temperature. The blots were incubated with primary antibodies in $3 \%$ BSA at $4{ }^{\circ} \mathrm{C}$ overnight, followed by the appropriate peroxidase-conjugated secondary antibodies for $60 \mathrm{~min}$ at room temperature. Membranes were developed with enhanced chemiluminescence reagent (Pierce Biosciences, USA) and visualized using G:BOX iChemi XR (Syngene, Cambridge, UK). Densitometry was performed using analysis software (GeneTools from SynGene).

\section{RT-PCR analysis}

Total RNA was extracted from the heart tissue using TRIzol reagent (Invitrogen, California, Carlsbad, USA) following the manufacturer's protocol. The concentration and purity of the RNA were determined using a Nanodrop 2000 (Thermo, USA). cDNA was synthesized with $2 \mu \mathrm{g}$ of RNA using a Hiscript II 1st Strand cDNA Synthesis Kit (Vazyme, Nanjing, China). The primer sequences for collagen I (176 bp) were as follows: forward: 5'-GCAATGCTGAATCGTCCCAC-3', reverse: 5'-CAGCACAGGCCCTCAAAAAC-3'. The primer sequences for collagen III (176 bp) were as follows: forward: 5'-TTCCTGGGAGAAATGGCGAC-3', reverse: 5'-CCAGGATAGCCACCCATTCC-3'. The primer sequences for GAPDH (451 bp) were as follows: forward: 5'-TGTGAACGGATTTGGCCGTA-3', reverse: 5'-TAAGCAGTTGGTGGTGCAGG-3'. cDNA was diluted 100 times with diethylpyrocarbonate (DEPC) water. The RT-PCR reaction was performed using a Biometra thermal cycler with $2 \times$ Taq Plus Master Mix (Vazyme, Nanjing, China). The PCR amplification was conducted as follows: $95^{\circ} \mathrm{C}$ for $5 \mathrm{~min}, 35$ cycles of $95^{\circ} \mathrm{C}$ for $30 \mathrm{~s}$ and $58^{\circ} \mathrm{C}$ or $60^{\circ} \mathrm{C}$ for $30 \mathrm{~s}$ and $72{ }^{\circ} \mathrm{C}$ for $45 \mathrm{~s}, 72{ }^{\circ} \mathrm{C}$ for $7 \mathrm{~min}$ and $4{ }^{\circ} \mathrm{C}$ ad infinitum. GAPDH was used as an endogenous control gene expressed constantly among samples. The products were detected using 1.5\% agarose gel electrophoresis and visualized using a Molecular Imager system (Bio-Rad, USA). The images were analyzed using Image Lab software.

\section{Statistical analysis}

Data are presented as the mean \pm SEM. Statistical analysis was performed using one-way analysis of variance (ANOVA) followed by Student-Newman-Keuls test using SPSS 10.0 statistical software. Differences were considered significant at $P<0.05$.

\section{Results}

Curcumin reduces the synthesis of collagen types I and III in the hearts of experimental diabetic rats

To examine the effect of curcumin on cardiac fibrosis, we conducted animal studies using normal and diabetic rats with or without curcumin treatment. As shown in Figure 1A and B, the protein and gene expression levels of collagen I and III in the myocardium of diabetic rats were significantly higher than those of the controls $(P<0.05)$ and were reduced in curcumintreated diabetic rats. Immunostaining for collagen I and III showed similar results (Figure 1C). The increased collagen synthesis was further confirmed by Masson trichrome staining. The blue area in the cardiac tissue, which indicated interstitial and perivascular collagen, was significantly increased in the diabetic group compared with the control and curcumintreated groups $(P<0.05)$.

\section{Curcumin inhibits TGF- $\beta 1$ production and Smad signaling in the hearts of experimental diabetic rats}

As TGF- $\beta 1$ has been identified as the most critical regulator in the pathogenesis of tissue fibrosis, we sought to detect TGF- $\beta 1$ signaling in STZ-induced experimental diabetic rats. Figure $2 \mathrm{~A}$ and $\mathrm{B}$ illustrate that TGF- $\beta 1$ production in diabetic hearts was significantly elevated compared with control hearts, and 
A

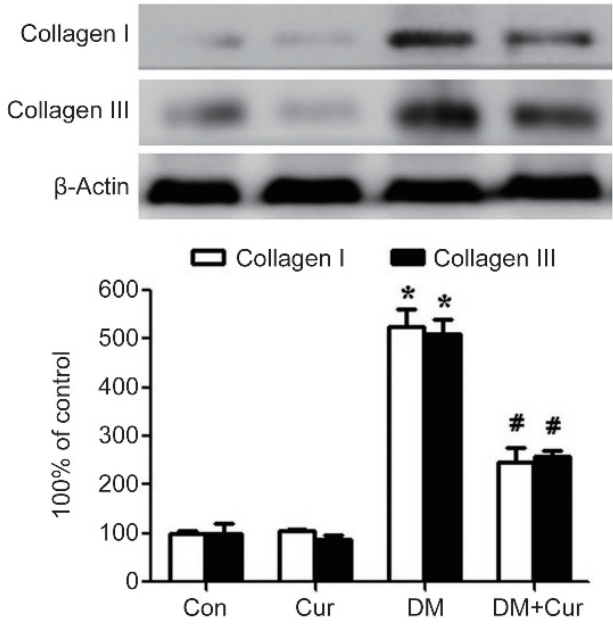

B

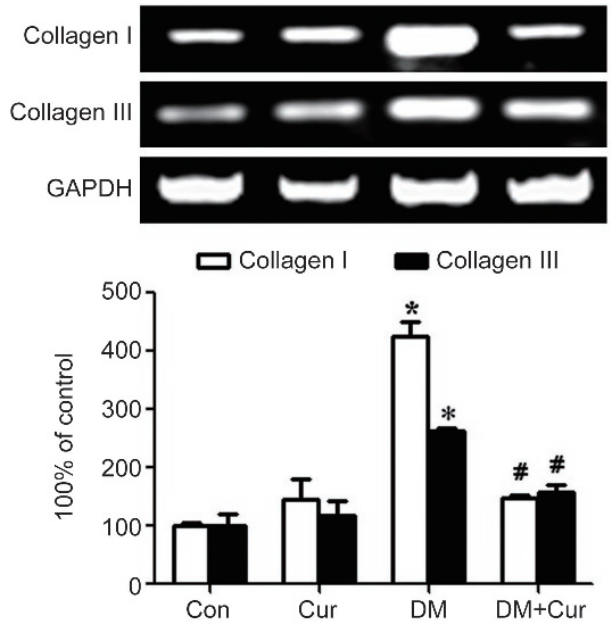

C
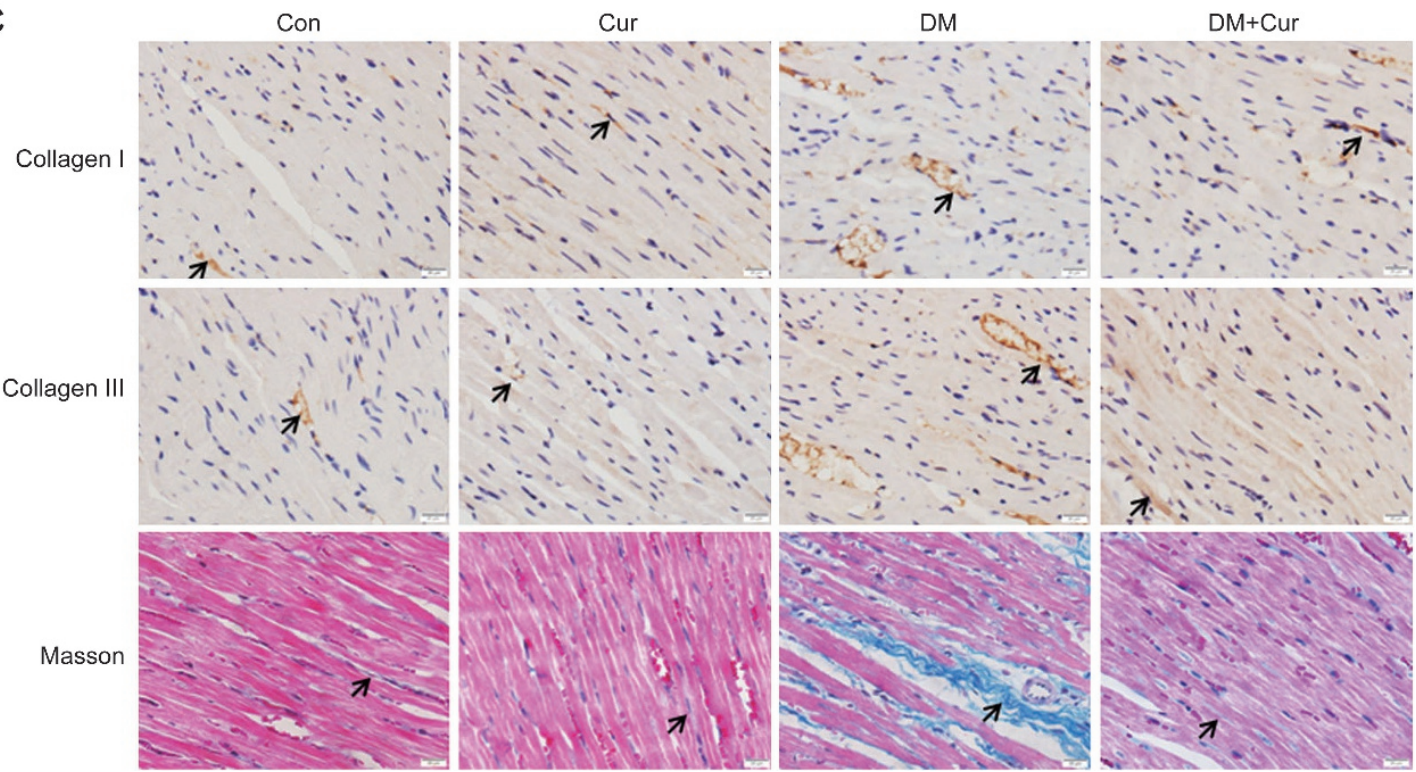

Figure 1. Curcumin decreases collagen synthesis in the hearts of experimental diabetic rats. A diabetic rat model was generated by low-dose STZ combined with high energy intake. Heart tissues were obtained from normal rats (Con group), normal rats treated with curcumin (Cur group), diabetic model rats (DM group), and diabetic model rats treated with curcumin (DM+Cur group). (A) The protein expression levels of collagen I and collagen III were measured by Western blotting. (B) The transcript levels of collagen I and collagen III were measured by PCR. (C) Immunostaining and Masson trichrome staining for collagen (magnification $=400 \times$, bar is $20 \mu \mathrm{m}$ ). The results are expressed as the mean \pm SEM. $n=6 /$ group. ${ }^{*} P<0.05$ vs control group. ${ }^{*} P<0.05$ vs DM group.

this elevation was attenuated in curcumin-treated diabetic rats $(P<0.05)$.

In canonical TGF- $\beta 1$ signaling, it is known that TGF$\beta 1$ exerts its effects via binding to the membrane-bound T $\beta R$ II, forming heteromeric complexes with the T $\beta R$ I and phosphorylating Smad2 and 3. The phosphorylated Smad2 and 3 then translocate into the nucleus to modulate the transcription of various genes, including those encoding collagen I and III. Smad7 acts as an inhibitory Smad to block this signal transduction by suppressing Smad2/3 phosphorylation. In parallel with the increased TGF- $\beta 1$ production, T $\beta R$ II expression was up-regulated in diabetic rats compared with control rats $(P<0.05$, Figure $3 \mathrm{~A})$. However, no significant difference was found among the four groups in terms of T $\beta R$ I level (Figure 3A). Additionally, Smad2/3 phosphorylation was enhanced, but Smad7 expression was reduced in diabetic rats compared with control rats. In addition, curcumin administration reversed these responses in diabetic rats (Figure 3B).

\section{Curcumin attenuates high glucose-induced collagen synthesis} and TGF- $\beta 1$ production in cardiac fibroblasts

As the main matrix-producing cells in the cardiac interstitium, fibroblasts are critically involved in all cardiac fibrotic conditions $^{[15]}$. To dissect the cellular effect of curcumin on cardiac fibrosis, we cultured human cardiac fibroblasts and exposed them to high glucose (D-glucose $30 \mathrm{mmol} / \mathrm{L})$ for 24 $\mathrm{h}$ with or without curcumin treatment. As shown in Figure 
A
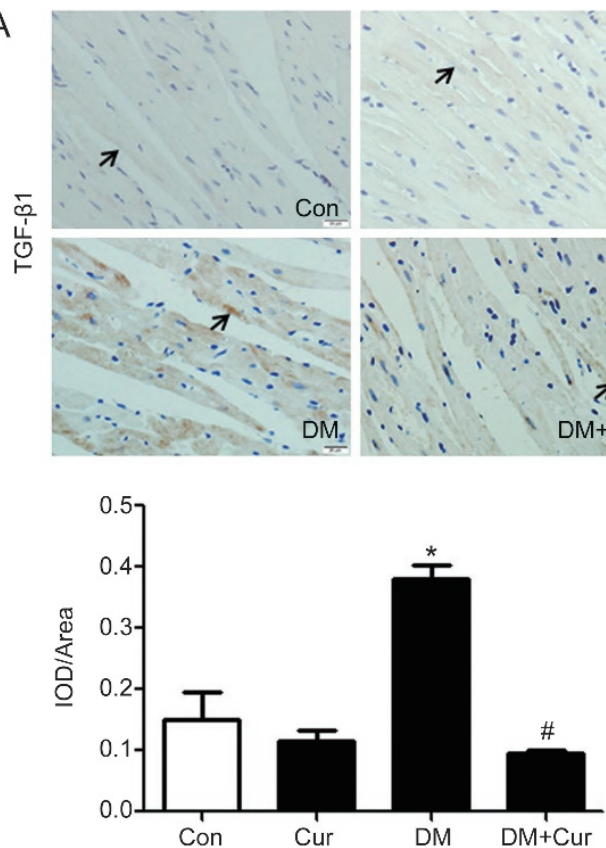

B
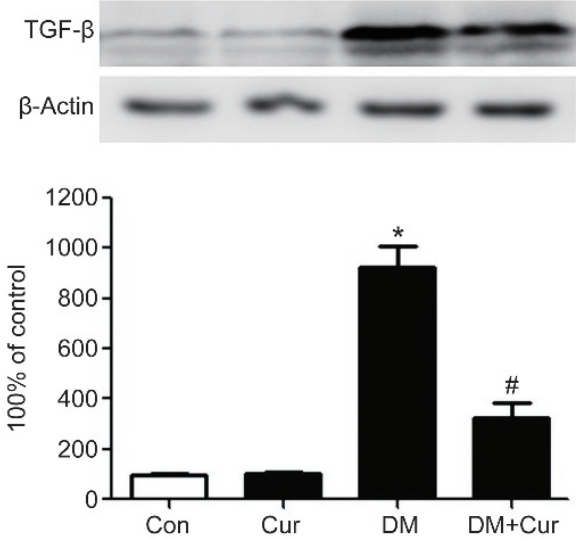

Figure 2. Curcumin inhibits TGF- $\beta 1$ production in the hearts of experimental diabetic rats. A diabetic rat model was generated by low-dose STZ combined with high energy intake. Heart tissues were obtained from normal rats (Con group), normal rats treated with curcumin (Cur group), diabetic model rats (DM group), and diabetic model rats treated with curcumin ( $D M+C u r$ group). (A) Representative immunostaining and quantitative analysis for TGF- $\beta 1$ expression ( $\times 400$, bar is $20 \mu \mathrm{m}$ ). (B) TGF- $\beta 1$ expression was measured by Western blotting. The results are expressed as the mean \pm SEM. $n=6$ /group. ${ }^{*} P<0.05$ vs control group. ${ }^{\#} P<0.05$ vs DM group.

$4 \mathrm{~A}$, the synthesis of collagen I and III was significantly increased by high glucose stimulation in contrast to normal glucose ( $D$-glucose $5.5 \mathrm{mmol} / \mathrm{L})$, and this effect was abrogated by curcumin treatment $(P<0.05)$. Figure $4 \mathrm{~B}$ also illustrates that raising the $D$-glucose concentration to $30 \mathrm{mmol} / \mathrm{L}$ for $24 \mathrm{~h}$ induced a significant increment in TGF- $\beta 1$ expression in
A

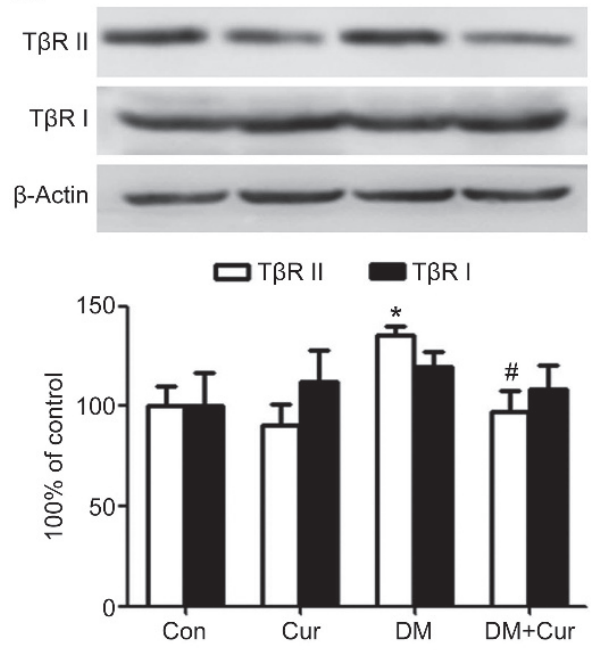

B

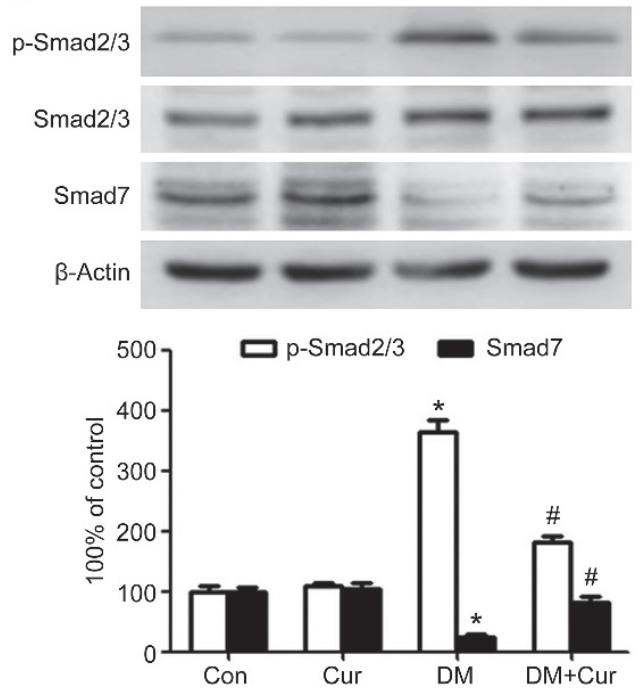

Figure 3. Curcumin inhibits the TGF- $\beta 1 /$ Smad signaling pathway in the hearts of experimental diabetic rats. A diabetic rat model was generated by lowdose STZ combined with high energy intake. Heart tissues were obtained from normal rats (Con group), normal rats treated with curcumin (Cur group), diabetic model rats (DM group), and diabetic model rats treated with curcumin (DM+Cur group). (A) Type II TGF- $\beta$ receptor (T $\beta R$ II) was down-regulated, but type I TGF- $\beta$ receptor (T $\beta$ R I) was little affected by curcumin treatment in the diabetic heart. (B) The phosphorylation of Smad2/3 was suppressed, whereas the reduction of Smad7 expression was reversed, by curcumin treatment in the diabetic heart. The results are expressed as the mean $\pm \mathrm{SEM}$. $n=6$ /group. ${ }^{*} P<0.05$ vs control group. ${ }^{\#} P<0.05$ vs DM group. 
A

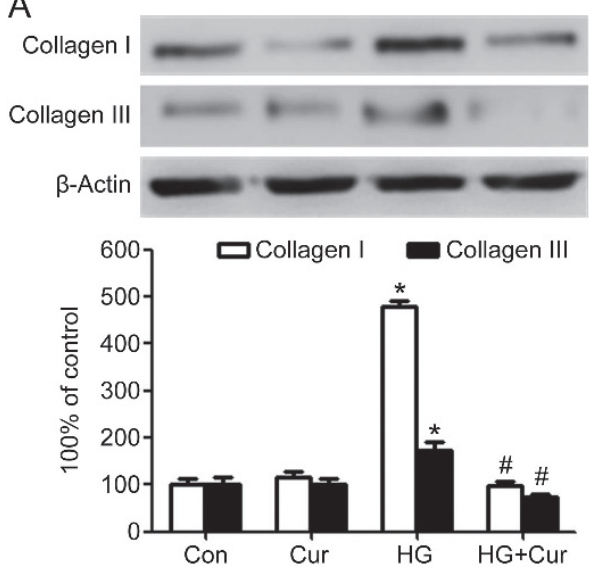

B
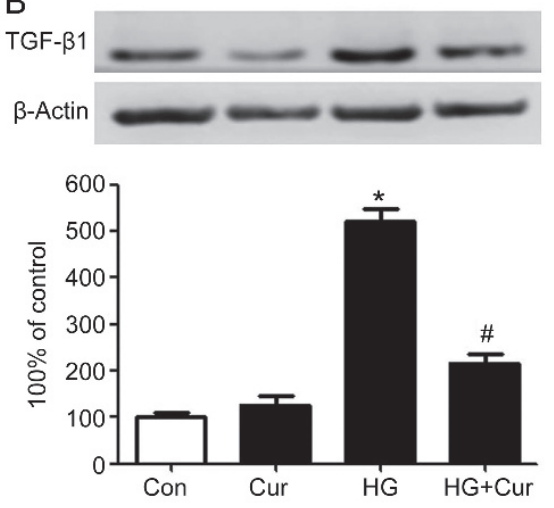

C

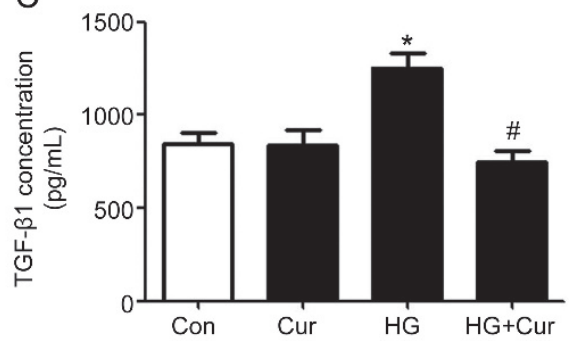

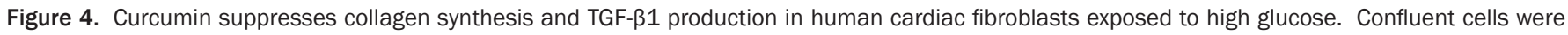

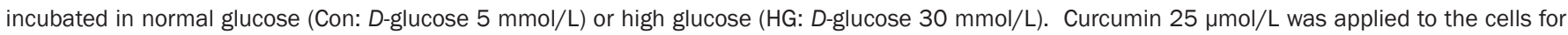

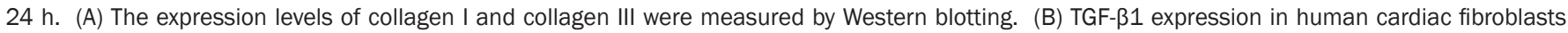

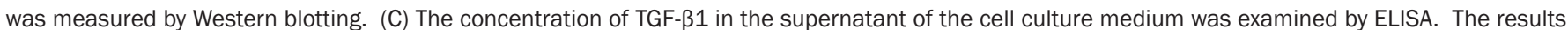

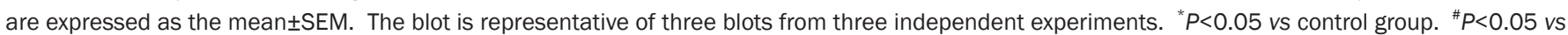
HG group.

human cardiac fibroblasts $(P<0.05)$. However, the addition of curcumin markedly inhibited TGF- $\beta 1$ production in cardiac fibroblasts subjected to high glucose. Curcumin also inhibited the secretion and release of TGF- $\beta 1$ from the cardiac fibroblasts, which we measured by examining the TGF- $\beta 1$ concentration in the supernatant of the cell culture medium usingELISA (Figure 4C).

\section{Curcumin inhibits high glucose-induced T $\beta R$ II/Smad signaling}

\section{activation in cardiac fibroblasts}

Consistent with the increased T $\beta R$ II/Smad signaling in the diabetic rat heart, enhanced expression of T $\beta R$ II and phosphorylation of Smad2/3 and inhibited Smad7 expression were detected in human cardiac fibroblasts incubated in $30 \mathrm{mmol} / \mathrm{L}$ high glucose $(P<0.05$, Figure $5 \mathrm{~A})$. By contrast, curcumin efficiently blocked high glucose-induced T $\beta \mathrm{R}$ II/Smad signaling activation $(P<0.05$, Figure $5 B)$.
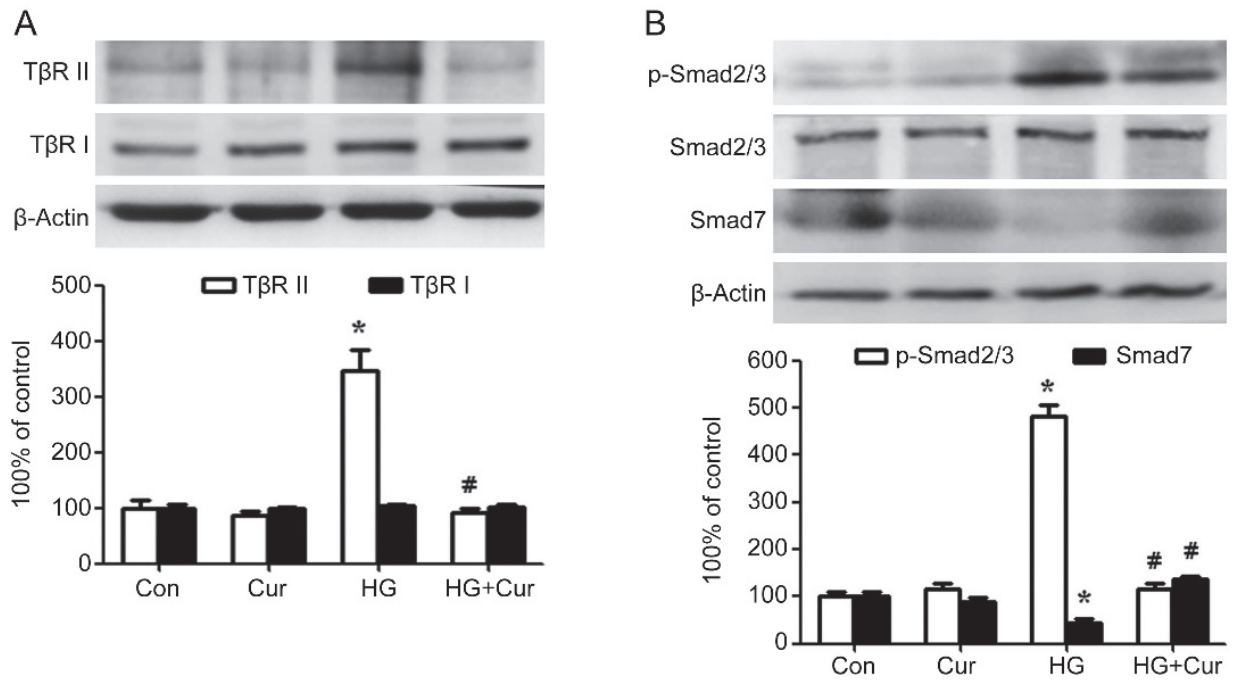

Figure 5. Curcumin inhibits the TGF- $\beta 1 /$ Smad signaling pathway in human cardiac fibroblasts exposed to high glucose. Confluent cells were incubated in normal glucose (Con: $D$-glucose $5 \mathrm{mmol} / \mathrm{L}$ ) or high glucose (HG: $D$-glucose $30 \mathrm{mmol} / \mathrm{L}$ ). Curcumin $25 \mu \mathrm{mol} / \mathrm{L}$ was applied to cells for $24 \mathrm{~h}$. (A) The expression of type II TGF- $\beta$ receptor (TRR II) was decreased, whereas the expression of type I TGF- $\beta$ receptor (T $\beta R$ I) was almost unchanged, in curcumintreated cardiac fibroblasts exposed to high glucose. (B) The phosphorylation of Smad2/3 was decreased, whereas the expression of Smad7 was restored, in curcumin-treated cardiac fibroblasts exposed to high glucose. The results are expressed as the mean \pm SEM. The blot is representative of three blots from three independent experiments. ${ }^{*} P<0.05$ vs control group. ${ }^{\#} P<0.05$ vs HG group. 
Curcumin blocks exogenous TGF- $\beta 1$-stimulated collagen synthesis and Smad2/3 signaling in cardiac fibroblasts

To determine whether curcumin has a direct effect on Smads or whether the regulation of $S \operatorname{mad} 2 / 3 / 7$ is just an adaptive response to reduced TGF- $\beta 1$ production, exogenous human recombinant TGF- $\beta 1$ ( $5 \mathrm{ng} / \mathrm{mL}$ ) was applied to cardiac fibroblasts for $24 \mathrm{~h}$. As shown in Figure 6A, the synthesis of collagen types I and III was significantly increased in response to TGF- $\beta 1$ stimulation $(P<0.05)$. Curcumin administration inhibited the expression of both collagen types $(P<0.05)$. Moreover, curcumin also significantly inhibited TGF- $\beta 1$-stimulated Smad2/3 phosphorylation $(P<0.05$, Figure $6 B)$. However, no significant difference was detected for curcumin in terms of TGF- $\beta 1$-stimulated Smad7 expression (Figure 6B).

The AMPK/p38 MAPK pathway is involved in collagen synthesis in cardiac fibroblasts exposed to TGF- $\beta$ or high glucose

In addition to the canonical pathway, TGF- $\beta$ can also activate the noncanonical p38 MAPK pathway to modulate its downstream signaling and related protein expression. Moreover, AMPK engages in cross-talk with the TGF- $\beta$ and p38 MAPK pathways in the cardiovascular system. The relationship among AMPK, p38 MAPK and collagen synthesis was examined in human cardiac fibroblasts stimulated by TGF- $\beta 1$ or high glucose. As depicted in Figure 7A and C, AMPK activity, which was assessed by monitoring the phosphorylation of AMPK at Thr172, was significantly enhanced when the cardiac fibroblasts were stimulated by $5 \mathrm{ng} / \mathrm{mL}$ TGF- $\beta 1$ or high glucose for $24 \mathrm{~h}(P<0.05$, Figure $7 \mathrm{~A}$ and $C)$. TGF- $\beta 1$ or high glucose stimulation also promoted the phosphorylation of p38 MAPK $(P<0.05$, Figure $7 A-C)$, accompanied by improved synthesis of collagen types I and III $(P<0.05$, Figure $7 \mathrm{~B}-\mathrm{D})$.

A

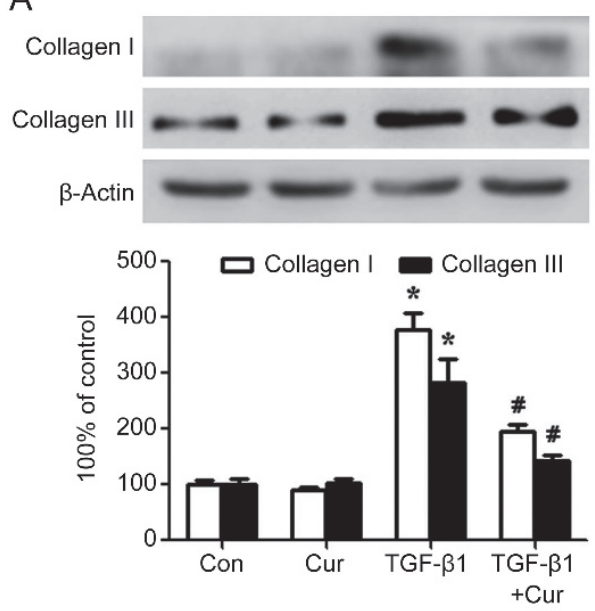

Curcumin treatment markedly abrogated the increased phosphorylation of AMPK and p38 MAPK, as well as the enhanced synthesis of collagen types I and III stimulated by TGF- $\beta 1$ or high glucose $(P<0.05$, Figure 7A-D). These effects were similar to the effects of Compound $\mathrm{C}$, a pharmacologic inhibitor of AMPK. By contrast, the AMPK activator metformin maintained not only the activity of AMPK and p38 MAPK, but also the collagen levels in cardiac fibroblasts exposed to TGF- $\beta 1$ or high glucose (Figure 7A-D). These results suggested that curcumin inhibited AMPK/p38MAPK activation in response to TGF- $\beta$ or high glucose in cardiac fibroblasts, which might help ameliorate cardiac fibrosis associated with diabetes.

\section{Discussion}

In this study, we have demonstrated that curcumin inhibits cardiac fibrosis associated with diabetes in vitro and in vivo. Our motivation for conducting these studies was related to our previous studies investigating the effects of curcumin on diabetic cardiomyopathy. We had found that curcumin orally administered at a dose of $300 \mathrm{mg} / \mathrm{kg}$ markedly mitigated interstitial fibrosis, myocardial apoptosis, and heart dysfunction in an experimental diabetic rat model. These phenomena were supported by studies from other laboratories reporting that curcumin not only inhibited cardiac fibrosis in response to angiotensin II (Ang II) and TGF- $\beta 1$ in myocardial infarction and cardiac pressure overload ${ }^{[16-19]}$, but also prevented multiple fibrotic diseases related to the lungs ${ }^{[20]}$, liver ${ }^{[21]}$, kidneys ${ }^{[22]}$, and oral submucosa ${ }^{[23]}$. Our present study extended this research to streptozotocin-induced experimental diabetic rats and demonstrated that increased gene transcription and protein synthesis of collagen types I and III in the heart were directly caused by the metabolic

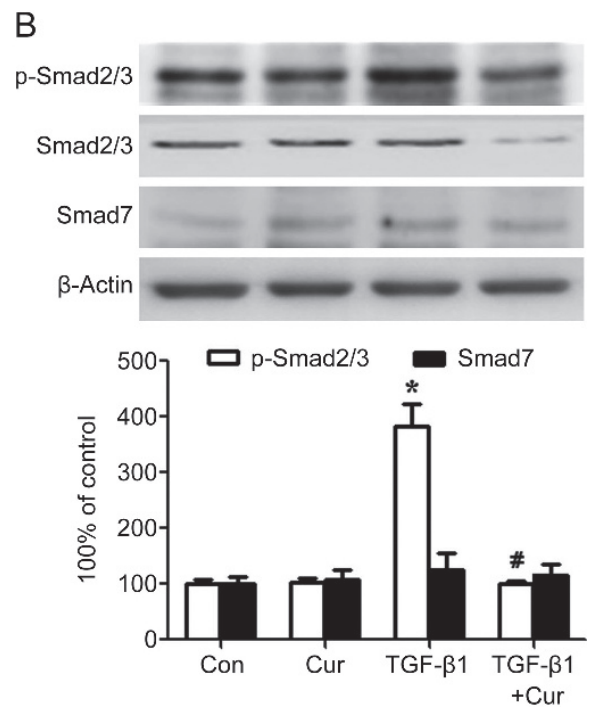

Figure 6. Curcumin blocks collagen synthesis and Smad2/3 phosphorylation stimulated by exogenous TGF- $\beta 1$ in human cardiac fibroblasts. Confluent cells were incubated in normal culture medium with the addition of $5 \mathrm{ng} / \mathrm{mL}$ exogenous human recombinant TGF- $\beta 1$. Curcumin (25 $\mu$ mol/L) was applied to cells for $24 \mathrm{~h}$. (A) Curcumin suppresses the synthesis of collagen I and collagen III induced in human cardiac fibroblasts by exogenous TGF- $\beta 1$. (B) Curcumin inhibits the phosphorylation of Smad2/3 in TGF- $\beta 1$-stimulated cardiac fibroblasts. The results are expressed as the mean $\pm S E M$. The blot is representative of three blots from three independent experiments. ${ }^{*} P<0.05$ vs control group. ${ }^{\#} P<0.05$ vs TGF- $\beta 1$ group. 

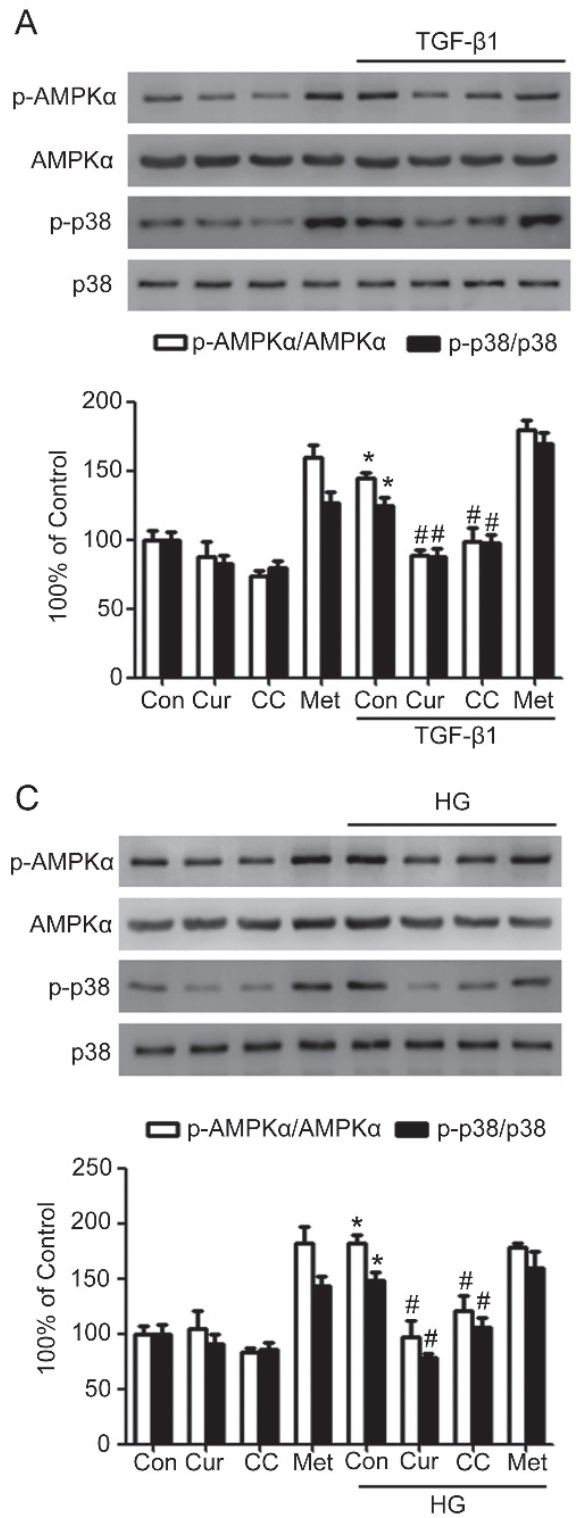

B

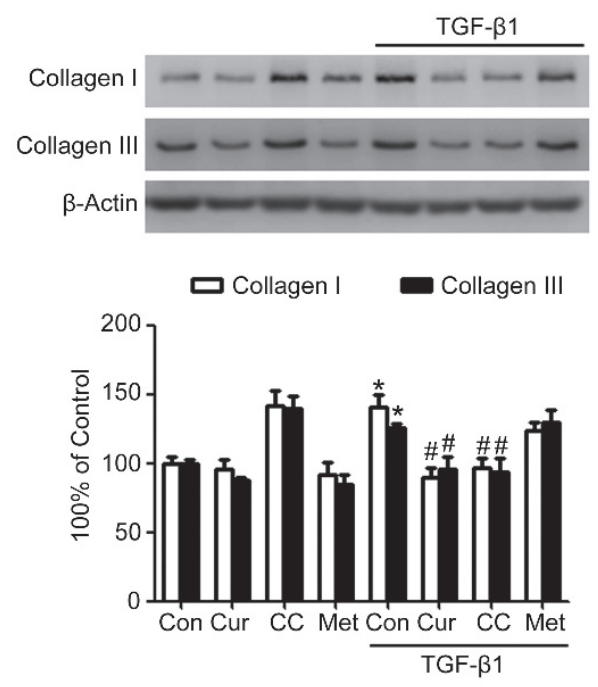

D
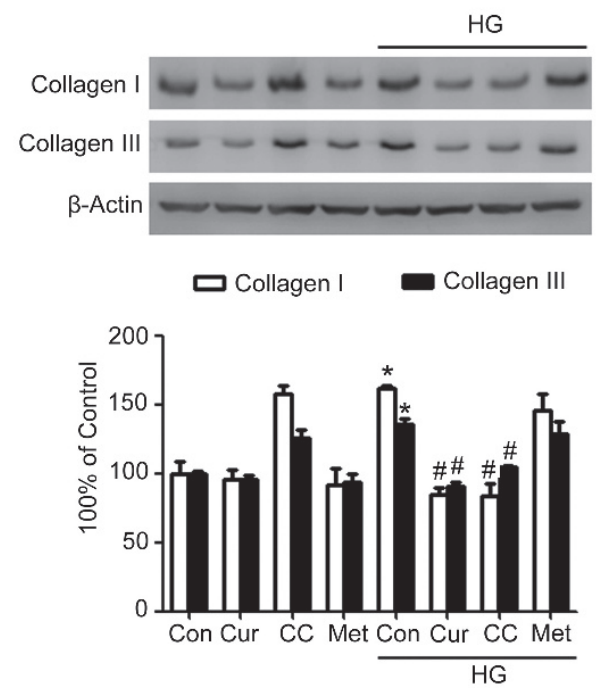

Figure 7. The AMPK/p38 MAPK pathway is involved in the inhibition of collagen synthesis by curcumin in cardiac fibroblasts exposed to TGF- $\beta$ or high glucose. Confluent cells were incubated in normal glucose (Con: $D$-glucose $5 \mathrm{mmol} / \mathrm{L}$ ), high glucose (HG: $D$-glucose $30 \mathrm{mmol} / \mathrm{L}$ ), or human recombinant TGF-B1 $(5 \mathrm{ng} / \mathrm{mL})$. Curcumin $(25 \mu \mathrm{mol} / \mathrm{L})$, Compound C (CC, $10 \mu \mathrm{mol} / \mathrm{L})$ or metformin (Met, $2 \mathrm{mmol} / \mathrm{L})$ was applied to cells for $24 \mathrm{~h}$. (A) Effects of curcumin, Compound $\mathrm{C}$ and metformin on the protein expression and phosphorylation of AMPK and p38 MAPK stimulated by TGF- $\beta 1$. (B) Effects of curcumin, Compound $\mathrm{C}$ and metformin on collagen synthesis stimulated by TGF- $\beta 1$. (C) Effects of curcumin, Compound $\mathrm{C}$ and metformin on the protein expression and phosphorylation of AMPK and p38 MAPK during exposure to high glucose. (D) Effects of curcumin, Compound C and metformin on collagen synthesis during exposure to high glucose. The results are expressed as the mean \pm SEM. The blot is representative of three blots from three independent experiments. ${ }^{*} P<0.05$ vs control group. ${ }^{*} P<0.05$ vs TGF- $\beta 1$ or HG group.

consequences of the diabetic state. The important role of hyperglycemia in the genesis of cardiac fibrosis was further supported by exposure of cardiac fibroblasts to high glucose levels. We demonstrated that curcumin treatment could suppress collagen synthesis both in an experimental animal model of diabetes mellitus and in cultured cardiac fibroblasts.

In general, myocardial collagen is divided into five types, namely, types I, III, IV, V, VI; among them, types I and III are the main components. Collagen type I is especially com- mon, accounting for almost $85 \%$ of the total collagen in heart, whereas type III accounts for $11 \%{ }^{[24]}$. The fiber of type I collagen is coarser so that it maintains the intensity of the ventricular wall, whereas type III collagen is much thinner to give better extension and elasticity. The synthesis and degradation of myocardial collagen are regulated by a complex network of growth factors and maintain a dynamic equilibrium state under physiological conditions. TGF- $\beta 1$ is considered as the most critical cytokine in the pathogenesis of tissue fibrosis ${ }^{[25]}$. 
Increased transcription, secretion, and activation of TGF- $\beta 1$ have been reported in experimental diabetic animals ${ }^{[26]}$. And in heart, TGF- $\beta 1$ may be generated by cardiomyocytes and a variety of non-cardiomyocytes, including macrophages, endothelial cells, and fibroblasts, in a paracrine or autocrine manner in vivo. Our present study provided direct evidence that TGF- $\beta 1$ production is increased in the diabetic heart. We further demonstrated that cardiac fibroblasts, the main matrixproducing cells, secreted and released more TGF- $\beta 1$ in the culture medium in response to high glucose in vitro ${ }^{[27]}$. Application of curcumin could significantly inhibit TGF- $\beta 1$ production both in the diabetic heart and in cultured cardiac fibroblasts. Thus, our study indicates that one likely explanation of how curcumin prevents diabetes-associated cardiac fibrosis is that it suppresses TGF- $\beta 1$ production in the myocardium.

TGF- $\beta 1$ is a pleiotropic cytokine that canonically acts through Smad signaling. Once activated, Smad2 and 3 translocate into the nucleus and stimulate the transcription of target genes, whereas Smad7 acts as an inhibitory component in this signal transduction pathway. We further demonstrated that curcumin directly inhibited Smad2/3 phosphorylation in cardiac fibroblasts in vitro. In addition, this effect was obviously more than an adaptive response to a lower TGF- $\beta 1$ concentration because we applied exogenous TGF- $\beta 1$ to the fibroblasts. Thus, in addition to decreasing TGF- $\beta 1$ production, curcumin also blocked the activation of canonical TGF- $\beta 1$ downstream signals in the diabetic state.

As a ubiquitously expressed cellular energy sensor, AMPK also plays an essential role in regulating cardiac fibrogenesis. The cross-talk between AMPK and the TGF- $\beta$ signaling pathway, the latter of which is critical in fibrosis, has been widely explored in previous studies. Transforming growth factor $\beta$-activated kinase 1 (TAK1), which is activated by a number of cytokines including TGF- $\beta$, was the third kinase identified as being upstream of AMPK in mammalian cells (in addition to LKB1 and CaMKK $\beta)^{[28]}$. TAK1 has also been described as an upstream kinase of $\mathrm{p} 38$ MAPK in the heart ${ }^{[29,30]}$. Thus, AMPK may be involved in regulating the non-canonical TGF- $\beta / \mathrm{p} 38$ MAPK pathway. In the present study, AMPK and p38 MAPK were activated in cultured cardiac fibroblasts in response to stimulation with TGF- $\beta 1$ or high glucose for $24 \mathrm{~h}$. This effect occurred in parallel with enhanced collagen synthesis. In the interest of identifying potential pharmacologic tools to manipulate AMPK, we further demonstrated that AMPK/p38 MAPK and collagen synthesis were inhibited by curcumin at this time point. In addition, these effects were similar to those of Compound C, a pharmacologic inhibitor of AMPK. By contrast, the AMPK activator metformin failed to further promote AMPK/p38 MAPK activity, but it maintained the level of collagen synthesis stimulated by TGF- $\beta 1$ or high glucose. Although most studies have suggested that AMPK activation inhibits fibrosis in various biological systems ${ }^{[31-32]}$, Cieslik et $a l^{[33]}$ reported that AICAR-dependent AMPK activation enhanced collagen synthesis as early as 5 days post occlusion in the ischemic heart. In addition, the elevated collagen deposition and scar contraction led to the formation of a function- ally competent scar that improves post-ischemic heart function and prevents adverse remodeling. A similar reparative fibrotic response may co-exist with interstitial and perivascular fibrosis in diabetic cardiomyopathy ${ }^{[34]}$. Diabetes and metabolic dysfunction may exert toxic effects on cardiomyocytes, eventually leading to irreversible injury and cell death. Fibrosis in diabetics may reflect replacement of dead cardiomyocytes with fibrous tissue ${ }^{[35]}$. We recognize that our study is limited in that we could not distinguish whether curcumin acts on reparative fibrosis or interstitial fibrosis at this time point. In addition, as AMPK activation might vary depending on the cellular context, the stimulation used and time points, we intend to follow up with more detailed analyses using different AMPK inhibitors (eg, siRNA) and different times of application. However, treatment of cardiac fibroblasts with curcumin for $24 \mathrm{~h}$ has been shown to inhibit high glucose- (or TGF- $\beta 1-$ ) induced collagen deposition at least partly by preventing AMPK activation. More studies are needed in the future to delineate the actions of AMPK in diabetes-associated cardiac fibrosis.

Taken together, the results of the present study demonstrated that curcumin prevents diabetes-associated collagen synthesis both in vivo and in vitro. This effect is attributable to inhibition of TGF- $\beta 1$ production and its downstream Smad-dependent and Smad-independent pathways. AMPK, at least in part, engages in cross-talk with TGF- $\beta /$ p 38 MAPK to mediate the inhibitory effect of curcumin on cardiac fibrosis.

\section{Acknowledgements}

This work was supported by grants from the Hubei Universities Outstanding Young Scientific and Technological Innovation Team Plans to Dr Chao LIU (№ T201213), from the New Century Excellent Talents Project of the Ministry of Education to Dr Chao LIU (No NCET-13-0781), and from Technology Innovation of Hubei Province (Major Projects) to Dr Chao LIU (No 2016ACA148).

\section{Author contribution}

The work presented was performed collaboratively by all authors. Chao LIU and Chang-han OU-YANG defined the research theme and designed the study; Shuang GUO, Xiangwen MENG and Xiu-fen LIU performed the research, analyzed the data and interpreted the results; Chao LIU and Xiao-song YANG wrote and revised the manuscript. All authors read and approved the final manuscript.

\section{References}

1 Travers JG, Kamal FA, Robbins J, Yutzey KE, Blaxall BC. Cardiac fibrosis: the fibroblast awakens. Circ Res 2016; 118: 1021-40.

2 Russo I, Frangogiannis NG. Diabetes-associated cardiac fibrosis: cellular effectors, molecular mechanisms and therapeutic opportunities. J Mol Cell Cardiol 2016; 90: 84-93.

3 Santibanez JF, Quintanilla M, Bernabeu C. TGF-beta/TGF-beta receptor system and its role in physiological and pathological conditions. Clin Sci (Lond) 2011; 121: 233-51.

4 Carling D. The AMP-activated protein kinase cascade-a unifying system for energy control. Trends Biochem Sci 2004; 29: 18-24.

5 Hawley SA, Davison M, Woods A, Davies SP, Beri RK, Carling D, et al. 
Characterization of the AMP-activated protein kinase kinase from rat liver and identification of threonine 172 as the major site at which it phosphorylates AMP-activated protein kinase. J Biol Chem 1996; 271: $27879-87$.

6 Decleves AE, Sharma K. Novel targets of antifibrotic and antiinflammatory treatment in CKD. Nat Rev Nephrol 2014; 10: 257-67.

7 Sato N, Takasaka N, Yoshida M, Tsubouchi K, Minagawa S, Araya $\mathrm{J}$, et al. Metformin attenuates lung fibrosis development via NOX4 suppression. Respir Res 2016; 17: 107.

8 Hallows KR, Fitch AC, Richardson CA, Reynolds PR, Clancy JP, Dagher $\mathrm{PC}$, et al. Up-regulation of AMP-activated kinase by dysfunctional cystic fibrosis transmembrane conductance regulator in cystic fibrosis airway epithelial cells mitigates excessive inflammation. J Biol Chem 2006; 281: 4231-41.

9 Daskalopoulos EP, Dufeys C, Bertrand L, Beauloye C, Horman S. AMPK in cardiac fibrosis and repair: actions beyond metabolic regulation. J Mol Cell Cardiol 2016; 91: 188-200.

10 Leu TH, Maa MC. The molecular mechanisms for the antitumorigenic effect of curcumin. Curr Med Chem Anticancer Agents 2002; 2: 357 70.

11 Jimenez-Osorio AS, Monroy A, Alavez S. Curcumin and insulin resistance-molecular targets and clinical evidences. Biofactors 2016; 42: 561-80.

12 Yu W, Wu J, Cai F, Xiang J, Zha W, Fan D, et al. Curcumin alleviates diabetic cardiomyopathy in experimental diabetic rats. PLoS One 2012; 7: e52013.

13 Shehzad A, Lee YS. Molecular mechanisms of curcumin action: signal transduction. Biofactors 2013; 39: 27-36.

14 Guo S, Yao Q, Ke Z, Chen H, Wu J, Liu C. Resveratrol attenuates high glucose-induced oxidative stress and cardiomyocyte apoptosis through AMPK. Mol Cell Endocrinol 2015; 412: 85-94.

15 Souders CA, Bowers SL, Baudino TA. Cardiac fibroblast: the renaissance cell. Circ Res 2009; 105: 1164-76.

16 Pang XF, Zhang LH, Bai F, Wang NP, Garner RE, McKallip RJ, et al. Attenuation of myocardial fibrosis with curcumin is mediated by modulating expression of angiotensin II AT1/AT2 receptors and ACE2 in rats. Drug Des Devel Ther 2015; 9: 6043-54.

17 Ma J, Ma SY, Ding $\mathrm{CH}$. Curcumin reduces cardiac fibrosis by inhibiting myofibroblast differentiation and decreasing transforming growth factor beta1 and matrix metalloproteinase 9/tissue inhibitor of metalloproteinase 1. Chin J Integr Med 2016; Doi:10.1007/S11655015-2159-5.

18 Liu H, Liu A, Shi C, Li B. Curcumin suppresses transforming growth factor-beta1-induced cardiac fibroblast differentiation via inhibition of Smad-2 and p38 MAPK signaling pathways. Exp Ther Med 2016; 11 : 998-1004.

19 Xiao J, Sheng X, Zhang X, Guo M, Ji X. Curcumin protects against myocardial infarction-induced cardiac fibrosis via SIRT1 activation in vivo and in vitro. Drug Des Devel Ther 2016; 10: 1267-77.

20 Tyagi N, Dash D, Singh R. Curcumin inhibits paraquat induced lung inflammation and fibrosis by extracellular matrix modifications in mouse model. Inflammopharmacology 2016; 24: 335-45.

21 Huang R, Liu Y, Xiong $\mathrm{Y}$, Wu H, Wang G, Sun Z, et al. Curcumin protects against liver fibrosis by attenuating infiltration of Gr1hi monocytes through inhibition of monocyte chemoattractant protein-1. Discov Med 2016; 21: 447-57.

$22 \mathrm{Hu} \mathrm{Y,} \mathrm{Mou} \mathrm{L,} \mathrm{Yang} \mathrm{F,} \mathrm{Tu} \mathrm{H,} \mathrm{Lin} \mathrm{W.} \mathrm{Curcumin} \mathrm{attenuates} \mathrm{cyclosporine}$ A induced renal fibrosis by inhibiting hypermethylation of the klotho promoter. Mol Med Rep 2016; 14: 3229-36.

23 Hazarey VK, Sakrikar AR, Ganvir SM. Efficacy of curcumin in the treatment for oral submucous fibrosis-a randomized clinical trial. J Oral Maxillofac Pathol 2015; 19: 145-52.

24 Jugdutt BI. Ventricular remodeling after infarction and the extracellular collagen matrix: when is enough enough? Circulation 2003; 108: 1395-403.

25 Biernacka A, Dobaczewski M, Frangogiannis NG. TGF-beta signaling in fibrosis. Growth Factors 2011; 29: 196-202.

26 Ziyadeh FN, Sharma K, Ericksen M, Wolf G. Stimulation of collagen gene expression and protein synthesis in murine mesangial cells by high glucose is mediated by autocrine activation of transforming growth factor-beta. J Clin Invest 1994; 93: 536-42.

27 Shinde AV, Frangogiannis NG. Fibroblasts in myocardial infarction: a role in inflammation and repair. J Mol Cell Cardiol 2014; 70: 74-82.

28 Momcilovic M, Hong SP, Carlson M. Mammalian TAK1 activates Snf1 protein kinase in yeast and phosphorylates AMP-activated protein kinase in vitro. J Biol Chem 2006; 281: 25336-43.

29 Biesemann N, Mendler L, Kostin S, Wietelmann A, Borchardt T, Braun T. Myostatin induces interstitial fibrosis in the heart via TAK1 and p38. Cell Tissue Res 2015; 361: 779-87.

30 Lu G, Kang YJ, Han J, Herschman HR, Stefani E, Wang Y. TAB1 modulates intracellular localization of p38 MAP kinase and downstream signaling. J Biol Chem 2006; 281: 6087-95.

31 Thakur S, Viswanadhapalli S, Kopp JB, Shi Q, Barnes JL, Block K, et al. Activation of AMP-activated protein kinase prevents TGFbeta1-induced epithelial-mesenchymal transition and myofibroblast activation. Am J Pathol 2015; 185: 2168-80.

32 Luo X, Deng L, Lamsal LP, Xu W, Xiang C, Cheng L. AMP-activated protein kinase alleviates extracellular matrix accumulation in high glucose-induced renal fibroblasts through mTOR signaling pathway. Cell Physiol Biochem 2015; 35: 191-200.

33 Cieslik KA, Taffet GE, Crawford JR, Trial J, Mejia Osuna P, Entman ML. AICAR-dependent AMPK activation improves scar formation in the aged heart in a murine model of reperfused myocardial infarction. J Mol Cell Cardiol 2013; 63: 26-36.

34 Turkbey EB, Backlund JY, Genuth S, Jain A, Miao C, Cleary PA, et al. Myocardial structure, function, and scar in patients with type I diabetes mellitus. Circulation 2011; 124: 1737-46.

35 Russo I, Frangogiannis NG. Diabetes-associated cardiac fibrosis: cellular effectors, molecular mechanisms and therapeutic opportunities. J Mol Cell Cardiol 2016; 90: 84-93. 